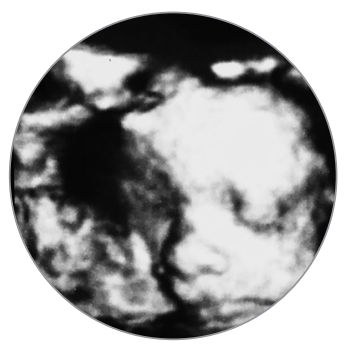

\section{Embarazo gemelar con mola hidatiforme completa y feto coexistente}

\author{
Twin Pregnancy with Complete Hydatidiform Mole and Coexistent \\ Fetus
}

\begin{tabular}{|l|}
\hline \\
Palabras clave (DeCS) \\
\hline $\begin{array}{l}\text { Enfermedad trofoblástica } \\
\text { gestacional } \\
\text { Embarazo gemelar } \\
\text { Ultrasonido }\end{array}$ \\
\hline Key words (MeSH) \\
\hline $\begin{array}{l}\text { Aneurysm } \\
\text { Gestational trophoblastic } \\
\text { disease } \\
\text { Twin pregnancy } \\
\text { Ultrasonics }\end{array}$ \\
\hline
\end{tabular}

'Residente de radiología, Universidad del Norte. Cartagena, Colombia.

${ }^{2}$ Ginecólogo obstetra, docente de ginecología y obstetricia, Universidad de Cartagena. Colombia.

Ecocenter. Cartagena, Colombia.

\title{
Resumen
}

Dentro del espectro de enfermedad trofoblástica gestacional, una de las entidades menos frecuente es la coexistencia, en un embarazo gemelar bicorial biamniótico, de una mola hidatiforme completa y un feto viable normal. El diagnóstico de esta condición requiere un cuidadoso examen ecográfico, con el fin de descartar la presencia de dos importantes patologías que componen los principales diagnósticos diferenciales en estos casos: la mola hidatiforme parcial y la displasia mesenquimal placentaria. Siguiendo de forma acuciosa el protocolo de ecografía obstétrica, y utilizando los conocimientos como explorador, se puede realizar el diagnóstico con precisión, diferenciando entre estas dos entidades. A continuación, se presenta el caso de un embarazo gemelar con mola hidatiforme completa y feto normal coexistentes, un hallazgo poco frecuente. Se realiza revisión de la literatura en cuanto a manejo y seguimiento de esta entidad dada su propensión a causar complicaciones en la gestación y a presentar comportamiento maligno.

\section{Summary}

Included in the spectrum of gestational trophoblastic disease, as one of the least frequent entities, is the coexistence, in a biamniotic twin pregnancy, of a complete hydatidiform mole and a normal viable fetus. The diagnosis of this condition requires a careful and thorough ultrasonographic examination, which will help in excluding the two main differential diagnosis in this scenario: partial hydatidiform mole and mesenchymal placental dysplasia. By following the obstetric examination protocol, and using academic radiologic knowledge, the examiner might reach a precise diagnosis. Here we present a case of a twin pregnancy with complete hydatidiform mole and coexistent fetus, a rare finding. We also present a short literature review on the topic of clinical management, given the fact that this disease might be associated with pregnancy complications and high risk of malignant behavior.

\section{Introducción}

Los embarazos gemelares con feto morfológicamente normal y mola hidatiforme coexistentes son un hallazgo infrecuente, con una incidencia estimada de 1 en cada 20.000 a 1 en cada 100.000 embarazos (1). Se diferencian de una mola parcial por un feto completo vivo de aspecto normal con su respectiva placenta, la cual se encuentra adyacente a una masa de tejido con cambios compatibles con mola hidatiforme (2). Se presenta un caso con hallazgos ecográficos claros documentados que muestran la coexistencia de un embarazo con feto normal y tejido placentario con evidentes cambios molares, confirmado por histopatología.

\section{Presentación del caso y hallazgos radiológicos}

Paciente de 19 años, primigestante, quien acude a urgencias por genitorragia moderada sin otra sintomatología asociada, normotensa. Al examen físico únicamente se encuentra como hallazgo positivo escasa genitorragia al tacto vaginal. Se documenta por ultrasonido un feto único vivo, de morfología normal, placenta de aspecto normal y localización fundocorporal con cordón de inserción marginal; adyacente a la placenta se describe - hacia la cara izquierda del cuerpo uterino - una masa de composición microquística, sin realce en el Doppler, que ocupa la cavidad uterina en su aspecto izquierdo, logrando delimitar sus contornos de forma clara con respecto a las capas de miometrio, al feto y al disco placentario normal descrito previamente (figuras 1, 2 y 3 ). Se concluye el estudio ecográfico como embarazo gemelar bicorial biamniótico, con gestación normal de 23 semanas 5 días y mola hidatiforme completa coexistente.

La paciente decide continuar la gestación y se hospitaliza para recibir control estricto por los especialistas tratantes. Al cumplir las 26 semanas de gestación, la paciente se torna hipertensa con perfil toxémico alterado, por lo cual se induce parto vaginal; sin embargo, por genitorragia abundante se le debe practicar cesárea. Se obtiene un feto único, vivo, sin malformaciones externas, unido al disco placentario por el cordón umbilical. Se extrae de la cavidad uterina material microquístico compatible con sospecha diagnóstica de mola hidatiforme. 


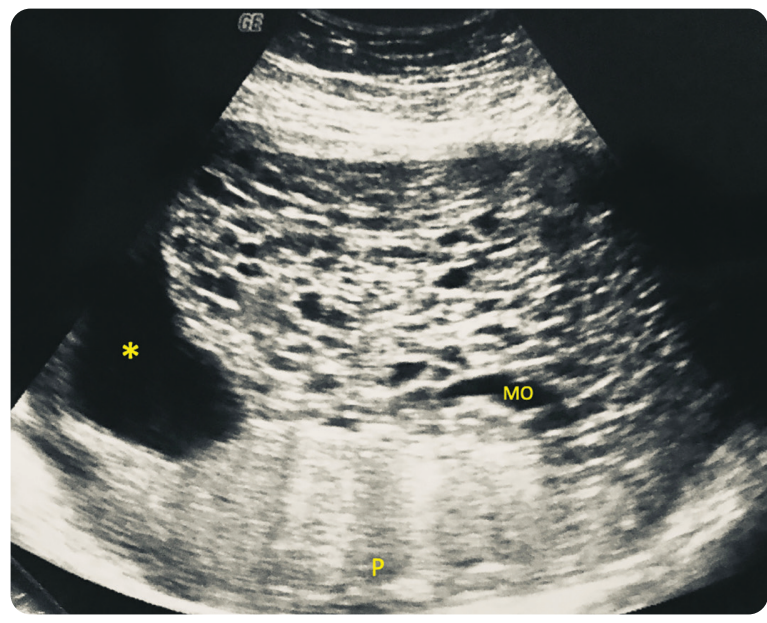

Figura 1. En esta imagen en orientación transversal se observan las siguientes estructuras: cavidad amniótica de la gestación viable (asterisco), gestación molar completa (MO) y disco placentario de la gestación viable (P). Feto no visible en este corte.

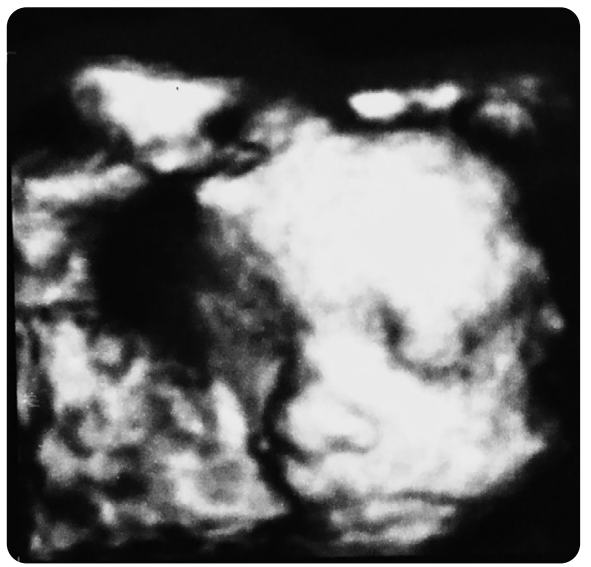

Figura 2. Imagen en 3D en la que se aprecia el rostro fetal sin alteraciones morfológicas.

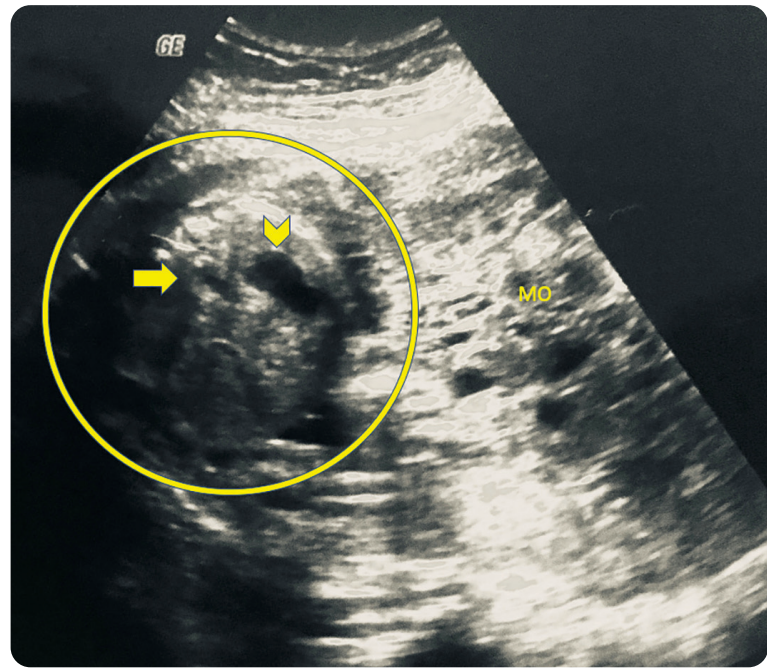

Figura 3. En esta imagen en orientación transversal se identifican las siguientes estructuras: señalado dentro del círculo se aprecia el abdomen fetal; la flecha, señala la aorta abdominal fetal, mientras que la cavidad gástrica se encuentra indicada por la cabeza de flecha. Adyacente a estas estructuras, a la derecha, la gestación molar (MO).
Se remiten el disco placentario, cordón y masa sospechosa de gestación molar para estudio anatomopatológico, el cual confirma que se trata de gestación bicorial biamniótica, con uno de los productos consistente en mola hidatiforme completa.

\section{Discusión}

La enfermedad trofoblástica gestacional consiste en un espectro de tumores malignos y benignos que incluye mola hidatiforme completa o parcial, mola infiltrante, coriocarcinoma, tumor trofoblástico del sitio de inserción placentaria y tumor trofoblástico epitelioide (3).

Se ha documentado alta variación en la prevalencia de la enfermedad trofoblástica dependiendo del país que informe: 1 por cada 1.000 embarazos en Estados Unidos y Europa. Los países asiáticos tienen la incidencia más alta de estos casos, lo cual se atribuye a grave deficiencia de vitamina A (4). Un gemelo sano puede desarrollarse de forma coexistente con una mola hidatiforme completa hasta en 1 de cada 20.000 embarazos, y hasta el $40 \%$ terminan en parto de producto vivo (3). El manejo de estos casos es un reto, debido al elevado riesgo de complicaciones prenatales y perinatales, que incluyen hemorragia anteparto y muerte fetal; es importante recalcar que hasta el $63 \%$ de las gestantes desarrollará enfermedad persistente posterior al parto, con metástasis en la mitad de los casos (4).

La coexistencia de mola hidatiforme completa y feto normal se puede dividir en tres tipos. El primero, correspondiente al descrito en este caso, consiste en una gestación gemelar en la que se presenta un feto normal - con cariotipo diploide (46 cromosomas, 23 maternos y 23 paternos) y con placenta de aspecto usual - acompañado por un gemelo consistente en una mola hidatiforme completa (46 cromosomas de origen paterno) (4). El segundo tipo resulta en un feto único de cariotipo triploide (69 cromosomas, 23 maternos y 46 paternos) acompañado de una placenta con cambios hidatiformes parciales (4). Por último, puede darse el caso de una gestación gemelar en la que uno de los productos es diploide con placenta normal (46 cromosomas, 23 maternos y 23 paternos) y el otro es triploide con mola hidatiforme parcial en la placenta (69 cromosomas, 23 maternos y 46 paternos) (4).

El ultrasonido sigue siendo la principal herramienta diagnóstica en las gestaciones molares parciales o completas (3). En este contexto, el componente molar se observará como múltiples espacios pequeños de contenido anecoico, los cuales varían de tamaño desde 1 a 30 $\mathrm{mm}$, lo cual se ha descrito como "racimo de uvas" y corresponden a vellosidades coriónicas hidrópicas (3). Dos diagnósticos diferenciales de la gestación gemelar con mola completa y feto normal coexistente consisten en la mola hidatiforme parcial y la displasia mesenquimal placentaria. En el primer caso, se presentan espacios quísticos en conjunto con feto fallecido morfológicamente alterado, mientras que la segunda entidad se caracteriza por la vascularización de los espacios quísticos; además, esta última consiste en una gestación monocorial, mientras que el embarazo gemelar con mola hidatiforme completa coexistente es dicigótico, en el que se identifican una placenta y feto normales, separadas de una placenta completamente molar, como es el caso en discusión (3).

Como se ha mencionado, existe riesgo elevado de complicaciones maternas y fetales en las gestaciones gemelares con una mola hidatiforme completa, incluyendo hemorragia vaginal, preeclampsia, hipertiroidismo, parto pretérmino y muerte fetal espontánea (5). Existe 
también, la probabilidad de enfermedad trofoblástica persistente/metastásica con requerimiento de quimioterapia, y son los pulmones el sitio más frecuente de metástasis, seguido por la vagina (5).

Anteriormente, el manejo de la gestación gemelar compuesto por mola hidatiforme completa y feto vivo consistía en la terminación temprana del embarazo $(5,6)$. Sin embargo, la continuidad de la gestación se ha convertido en una opción viable en el siglo XXI, apoyada por una serie de casos japoneses en la que los investigadores notaron que el riesgo de malignidad no cambia con la edad gestacional avanzada, pero es importante la adecuada monitorización de complicaciones (7).

En el contexto de la enfermedad trofoblástica gestacional maligna, se ha descrito que esta es altamente sensible a la quimioterapia y se cuenta con varios esquemas de manejo $(5,8)$. En etapa temprana, esta entidad se cura con frecuencia usando un único agente quimioterapéutico, mientras que en etapas avanzadas se requiere de varios agentes para lograr dicho desenlace, entre ellos la combinación de etopósido, metotrexate, actinomicina $\mathrm{D}$, ciclofosfamida y vincristina es la que más se usa, con remisión completa en un $78 \%$ de los casos $(5,8)$.

El caso descrito cobra importancia por la riqueza de sus hallazgos imagenológicos y su poca frecuencia; es importante la adecuada visualización de todas las partes fetales, la presencia del cordón umbilical unido a una placenta de aspecto ecográfico usual y su coexistencia con una mola completa que, ecográficamente, logra separarse de la gestación viable.

Desde el punto de vista clínico, es de anotar que estas gestaciones pueden llegar a término dependiendo de las comorbilidades maternas, el bienestar fetal y de la calidad del seguimiento que se pueda ofrecer. Es importante también el seguimiento clínico al finalizar la gestación y en embarazos siguientes, dado el riesgo de enfermedad metastásica y recurrencia de gestación molar.

\section{Referencias}

1. Steller M, Genest DR, Bernstein MR, et al. Natural history of twin pregnancy with complete hydatidiform mole and coexisting fetus. Obstet Gynecol. 1994;83:35-42.

2. Lazarus E, Levine D. The first trimester, diagnostic ultrasound, quinta edición. Capítulo 30. 2018. pp. 1048-1087.

3. Shaaban A, Rezvani M, Haroune M, et al. Gestational trophoblastic disease: Clinical and imaging features. RadioGraphics. 2017;37:681-700.

4. Lipi LB, Philp L, Goodman AK. A challenging case of twin pregnancy with complete hydatidiform mole and co-existing normal live fetus - A case report and review of the literature. Gynecologic Oncology Reports. 2020;31:100519.

5. Peng HH, Huang KG, Chue HY, et al. Term delivery of a complete hydatidiform mole with a coexisting living fetus followed by successful treatment of maternal metastatic gestational trophoblastic disease. Taiwanese J Obstet Gynecol. 2014;53:397-400.

6. Nobuhara I, Harada N, Haruta N, et al. Multiple metastatic gestational trophoblastic disease after a twin pregnancy with complete hydatidiform mole and coexisting fetus, following assisted reproductive technology: Case report and literature review. Taiwanese J Obstet Gynecol. 2018;57:588-93.

7. Matsui H, Sekiya S, Hando T, Wake N, Tomoda Y. Hydatidiform mole coexis- tent with a twin live fetus: a national collaborative study in Japan. Humanit Rep. 2000;15:608-11.

8. Deng L, Yan X, Zhang J, Wu T. Combination chemotherapy for high-risk gestational trophoblastic tumour. Cochrane Database Syst Rev. 2009;15:CD005196.

\section{Correspondencia}

César Enrique Mendivil Silva

Carrera 52 \# 82-278

Barranquilla, Colombia

mendivilc@uninorte.edu.co

Recibido para evaluación: 20 de abril de 2020

Aceptado para publicación: 8 de mayo de 2020 